\title{
A natural supplement formula reduces anti-oxidative stress and enhances osteo-chondrogenic differentiation potential in mesenchymal stem cells
}

\author{
Dong Hyun Kim, ${ }^{1,2,+}$ Dong Hwan Kim, ${ }^{3,+}$ Bruce E. Heck, ${ }^{2}$ Michael Shaffer, ${ }^{2}$ Jin Hur ${ }^{4, *}$ and Keon Hee Yoo ${ }^{1, *}$ \\ ${ }^{1}$ Department of Pediatrics, Hematology/Oncology, Samsung Medical Center, Sungkyunkwan University School of Medicine, 50 Irwon-dong, Gangnam-gu, \\ Seoul 06351, Republic of Korea \\ ${ }^{2}$ NWO Stem Cure, LLC, 7595 CR 236, Findlay, OH 45840, USA \\ ${ }^{3}$ Department of Orthopedic Surgery, Seoul National University Hospital, 101, Daehak-ro, Jongno-ku, Seoul 03080, Republic of Korea \\ ${ }^{4}$ Department of Convergence Medicine, Pusan National University School of Medicine, 49 Busandaehak-ro, Yangsan, Gyeongsangnam-do 50612, Republic of Korea
}

There is great interest in using natural supplements to treat various medical conditions. In this study, we evaluated the antioxidative and stem cell differentiation effects of a mixture of vitamin D, Lactobacillus rhamnosus, ginger, curcumin, and Boswellia extract. The calcein acetoxymethyl assay after $\mathrm{H}_{2} \mathrm{O}_{2}$ treatment showed that combined natural supplement had an anti-oxidative effect. NS-J also increased calcium deposition, as shown by Alizarin Red S staining, indicating bone formation activity. The contents of type II collagen and glycosaminoglycans, which are biomarkers of cartilage, were higher in mesenchymal stem cells treated with combined natural supplement than in cells treated with individual ingredients of the formula. In mesenchymal stem cells treated with human osteoarthritis synovial fluids, combined natural supplement enhanced the expression of type II collagen and PPAR- $\delta$, overcoming the anti-chondrogenic effect of inflammatory conditions. Combined natural supplement also inhibited Oil Red $O$ staining in cells, which indicates inhibited adipogenesis. Thus, combined natural supplement, a formula comprising vitamin $D$, Lactobacillus rhamnosus, ginger, curcumin and Boswellia extract, reduced oxidative stress, enhanced osteogenesis and chondrogenesis, and inhibited adipogenesis in mesenchymal stem cells to a greater extent than the individual ingredients, indicating synergistic interaction. In addition, combined natural supplement increased the expression PPAR- $\delta$, suggesting that these effects correlate with the PPAR- $\delta$ pathway.

Key Words: natural ingredients, osteogenesis, adipogenesis, chondrogenesis

$\mathrm{T}$ he use of natural supplements has a long history of treating multiple diseases. ${ }^{(1)}$ In our experience, these supplements have various effects such as pain reduction and anti-inflammation. ${ }^{(1)}$ Since natural supplements are complex mixtures, their net efficacy results from multiple interactions among the constituents. ${ }^{(2-4)}$ Effects of natural dietary supplements have been published in various fields, such as their ability to improve in mitochondrial dysfunction. ${ }^{(5)}$ Interest in natural supplements is growing, and many relevant studies are ongoing around the world. Vitamin D is registered with the Food and Drug Administration (FDA) and has various positive effects on humans, such as improving knee cartilage integrity, ${ }^{(6)}$ suppressing arthritic inflammation, ${ }^{(7)}$ and increasing bone density. ${ }^{(8)}$ Lactobacillus rhamnosus (L. rhamnosus), also registered with the FDA, has been reported to improve arthritis ${ }^{(9)}$ and inflammation $^{(10,11)}$ and reduce tissue damage, ${ }^{(12)}$ oxidative stress, ${ }^{(13)}$ and adiposity. ${ }^{(14)}$ Ginger has a strong ability to reduce the pain and other symptoms of arthritis. ${ }^{(15)}$ It also has anti-oxidative and anti-inflammatory effects. ${ }^{(16)}$ Curcumin has anti-arthritis ${ }^{(17)}$ and anti-inflammatory effects. ${ }^{(18)}$ Curcumin also enhances cartilage formation $^{(19)}$ and muscle repair. ${ }^{(20)}$ Boswellia plant extract has anti-arthritic, ${ }^{(21)}$ analgesic, ${ }^{(22)}$ and anti-inflammatory effects. ${ }^{(23)}$ Thus, all those natural products have individually contributed significantly to the well-being of people.

Several papers have reported that those natural supplements show synergism. ${ }^{(2-4)}$ Jerzynska et al. ${ }^{(3)}$ reported that co-administration of vitamin D supplements and Lactobacillus rhamnosus showed better immunological response than individual administration in pediatric allergy rhinitis patients. These natural supplements show similar properties, but combining them can generate additive or synergistic effects on the human body. However, research on the mechanisms of natural supplements remain inadequate. Also, further research is needed on how natural supplements or supplement combinations influence stem cells.

Peroxisome proliferator-activated receptors (PPARs) are members of the nuclear hormone receptor superfamily and play an important role in glucose and fat metabolism. ${ }^{(24)}$ PPAR- $\delta$ is expressed ubiquitously and involved in apoptosis, fat metabolism and chondrogenesis. ${ }^{(25)}$ Heck et al. ${ }^{(25)}$ reported that a PPAR- $\delta$ selective agonist (GW0742) had enough chondrogenic effect to overcome the anti-chondrogenic effect of inflammation. The previously mentioned natural supplements are all anti-inflammatory and pro-chondrogenic. A comparative study of a mixture of vitamin D, L. rhamnosus, ginger, curcumin, and Boswellia extract (NS-J) and PPAR- $\delta$ is thus a useful way to investigate the mechanism of those natural supplements.

For this study, we investigated how the combination of these natural ingredients would affect mesenchymal stem cells (MSCs) in terms of resistance to oxidative stress and differentiation potential. We also demonstrate a correlation between PPAR- $\delta$ and NS-J.

\section{Methods}

Isolation and culture of hBM-MSCs. Frozen mononuclear cells from human bone marrow were purchased (AllCells,

${ }^{\dagger}$ These authors contributed equally to this work.

*To whom correspondence should be addressed.

E-mail: gene44@pusan.ac.kr (JH); hema2170@skku.edu (KHY) 
Emeryville, CA) and used to isolate human bone marrowmesenchymal stem cells (hBM-MSCs). Briefly, thawed mononuclear cells were cultured in Dulbecco's modified Eagle medium (DMEM) plus 20\% fetal bovine serum (FBS) and 1\% antibioticantimycotic solution (Invitrogen, Carlsbad, CA) and plated at a density of $1 \times 10^{6}$ cells $/ 75-\mathrm{cm}^{2}$ flask. Adherent cells were re-plated at a density of $1-5 \times 10^{6}$ cells per $75-\mathrm{cm}^{2}$ flask and cultured at $37^{\circ} \mathrm{C}$ in a $5 \% \mathrm{CO}_{2}$ incubator. The culture medium was exchanged with fresh medium after $48 \mathrm{~h}$ and then every 3-4 days until the cells were $70 \%$ confluent.

Extract preparation and NS-J. Vitamin D, L. rhamnosus, ginger, curcumin and Boswellia extract powder were supplied by Pure Source, LLC (Doral, FL). Dried samples were blended to a fine powder and extracted three times $(30 \mathrm{~min}$ each with continuous stirring at $60^{\circ} \mathrm{C}$ ) with dimethyl sulfoxide (DMSO). The filtrates were combined and concentrated using a rotary evaporator. The collected samples were kept in amber colored screw capped bottles at $-18^{\circ} \mathrm{C}$ until analysis. Stock solutions in DMSO were prepared at concentrations of $50 \mathrm{mg} / \mathrm{ml}$. Formula NS-J contained vitamin D3, L. rhamnosus, ginger root extract, curcumin from turmeric root extract and Boswellia serrata extract. The concentration of NS-J is $50 \mu \mathrm{g} / \mathrm{ml}$ and the ratio of each natural ingredients are 1:12:91:64:91.

Hydrogen peroxide treatment to induce oxidative stress. hBM-MSCs (passages 2-3) were re-plated on a six well-plate and incubated in DMEM plus $10 \%$ FBS and $1 \%$ antibiotic-antimycotic solution for 1 day. Then, the hBM-MSCs were treated with $50 \mu \mathrm{g} / \mathrm{ml}$ of vitamin D, L. rhamnosus, ginger, curcumin, Boswellia extract, or NS-J. hBM-MSCs (passage 6) were seeded in the wells of 24-well plates at a density of $1 \times 10^{5} \mathrm{cells} / \mathrm{cm}^{2}$ and treated with $100 \mu \mathrm{M} \mathrm{H}_{2} \mathrm{O}_{2}$. MSCs were incubated in $1 \times$ PBS containing $2 \mu \mathrm{M}$ calcein acetoxymethyl ester (calcein-AM, live cells: green) and $4 \mu \mathrm{M}$ ethidium homodimer-1 (dead cells: red) for $10 \mathrm{~min}$ at $37^{\circ} \mathrm{C}$. Immediately thereafter, the cells were imaged under the $10 \times$ objective of a fluorescence Olympus IX71 microscope (Olympus America Inc., Center Valley, PA). Five thousand cells per treatment were analyzed for viability using Metamorph software (Molecular Devices, Inc., Downingtown, PA).

Osteogenic differentiation of human MSCs. hBM-MSCs (passages 2-3) were re-plated in a $75-\mathrm{cm}^{2}$ flask at a density of $1 \times 10^{5}$ cells $/ \mathrm{cm}^{2}$ and cultured in the alpha modification of minimum essential medium ( $\alpha$-MEM) with $20 \% \mathrm{FBS}$ at $37^{\circ} \mathrm{C}$ for 1 day. The next day, the medium was switched to Stem X-Vivo medium (R\&D System, Minneapolis, MN). MSCs were cultured in osteogenic medium with and without $50 \mu \mathrm{g} / \mathrm{ml}$ of vitamin D, L. rhamnosus, ginger, curcumin, Boswellia extract, or NS-J. The medium was replaced every other day for 21 days. To assess calcium deposition, the cells were washed with ice-cold PBS and incubated in $10 \%$ paraformaldehyde for $10 \mathrm{~min}$. Then, the cells were incubated in $2 \%$ Alizarin Red S (Sigma, St. Louis, MO), which stains calcium in cells, at $\mathrm{pH} 4.2$ for $10 \mathrm{~min}$ and washed three times with distilled water. The stained cells were visualized by phase-contrast microscopy to determine cell morphology and verify the presence of mineralized nodules (red) after image acquisition. For quantification, an Osteogenesis Quantitation Kit (Millipore, Billerica, MA) was used following the manufacturer's recommendation. Briefly, $400 \mu \mathrm{l}$ of $10 \%$ acetic acid was added to each well of a 24-well plate. Cells were incubated for $30 \mathrm{~min}$ on a shaker, gently scraped off the plate, and transferred to a $1.5-\mathrm{ml}$ microtube. After vigorous vortexing for $30 \mathrm{~s}$, the samples were heated to $85^{\circ} \mathrm{C}$ for $10 \mathrm{~min}$ and incubated on ice for $5 \mathrm{~min}$. After centrifugation at $20,000 \times g$ for $15 \mathrm{~min}$, the supernatant was transferred to a new $1.5-\mathrm{ml}$ microtube. The acidic $\mathrm{pH}$ of the sample was neutralized with ammonium hydroxide. The optical density of the sample was measured at $405 \mathrm{~nm}$ using a microplate reader.

Chondrogenic differentiation of human MSCs. Chondrogenic differentiation was assessed by high-density micromass culturing on hyaluronic acid (HA) gel (Euflexxa ${ }^{\circledR}$; Ferring Pharmaceuticals Inc., Parsippany, NJ). hBM-MSCs (passages 2-3) were plated in a $75-\mathrm{cm}^{2}$ flask at a density of $1.5 \times 10^{6}$ cells $/ \mathrm{cm}^{2}$ and cultured in $\alpha$-MEM with $20 \%$ FBS for 1 day at $37^{\circ} \mathrm{C}$. The next day, the hBM-MSCs were harvested and re-plated on $70 \mu \mathrm{l}$ of HA gel (Euflexxa $\left.{ }^{\circledR}\right)$ at a density of $8 \times 10^{6}$ viable cells/well in a 96well plate. Then, $50 \mu$ l of chondrogenesis medium (STEMPRO ${ }^{\mathbb{R}}$ Chondrogenesis Differentiation Kit, Life Technologies, Grand Island, NY) was added to each well with and without vitamin D, L. rhamnosus, ginger, curcumin, Boswellia extract, or NS-J. After chondrocyte spheroids formed, the culture medium was replaced with fresh chondrogenesis medium every 2 days for the next $14-$ days of incubation for chondrogenesis.

Chondrogenic differentiation of human MSCs with human OA synovial fluids. Human osteoarthritis (OA) synovial fluid was used to assess the effects of inflammation. hBM-MSCs (passage 2-3) were incubated in chondrogenic medium with and without HA, NS-J and 50\% human OA synovial fluid for 14 days. By the 14th day, chondrocyte spheroids had formed, and images of them were captured using a light microscope.

Then, the chondrocytes were processed for immunoblotting using antibodies to type II collagen $(1: 1,000$ dilution; EMD Millipore, Billerica, MA), PPAR- $\delta$ (1:2,000 dilution; Abcam, Cambridge, MA) and $\beta$-actin as a loading control $(1: 10,000$ dilution; Abcam). Secondary antibodies tagged with horseradish peroxidase (mouse for type II collagen and $\beta$-actin, 1:5,000 dilution) were used. The proteins were separated by polyacrylamide gel electrophoresis and then transferred to nitrocellulose membranes (GE Healthcare Life Sciences, Pittsburgh, PA) using a TE77XP semidry blotter operated at $10 \mathrm{~V}$ for $3 \mathrm{~h}$ (Hoefer, Inc., Holliston, MA). Protein band signals on the blots were detected using Amersham Hyperfilm with enhanced chemiluminescence sensitivity (GE Healthcare Life Sciences) and a SuperSignal West Pico Chemiluminescent substrate kit (Thermo Fisher Scientific, Rockford, IL).

The use of human synovial fluid was approved by the Institutional Review Board of University of Toledo. Discarded synovial fluids were obtained after receiving informed consent from patients.

Adipogenic differentiation of human MSCs. hBM-MSCs (passages 2-3) were incubated in adipogenic medium (DMEM containing high glucose, $4.5 \mathrm{~g} / \mathrm{L}$; Invitrogen), $10 \mu \mathrm{g} / \mathrm{ml}$ insulin, $60 \mu \mathrm{M}$ indomethacin, $10 \% \mathrm{FBS}$, and antibiotic-antimycotic (Invitrogen) and treated with $50 \mu \mathrm{g} / \mathrm{ml} \mathrm{NS}-\mathrm{J}$ every second day for 14 days. On the 14th day, the cells were stained with Oil Red O, which stains lipid droplets, and imaged using a light microscope. Lipid droplets were eluted by adding $100 \mu \mathrm{l}$ of isopropanol to each well. The absorbance of the elute was measured at $490 \mathrm{~nm}$ using a microplate reader to evaluate adipogenesis.

\section{Results}

NS-J protects hBM-MSCs from oxidative stress. We performed a live/dead cell analysis using calcein AM (live cells: green) and ethidium homodimer-1 (dead cells: red). Most untreated MSCs underwent apoptosis after a 12-h exposure to $\mathrm{H}_{2} \mathrm{O}_{2}$ (Fig. 1A). MSCs treated with ginger and curcumin were protected against oxidative stress. Conversely, those treated with vitamin D and Boswellia extract did not show any difference in survival from control cells. We then tested whether the combination of vitamin D, L. rhamnosus, ginger, curcumin, and Boswellia extract protected MSCs from oxidative stress. The combination significantly increased the percentage of surviving MSCs by 10fold compared with the control (Fig. 1B). This result suggests that natural supplements, especially ginger and curcumin protect MSCs from oxidative stress, and that the individual components of NS-J interacted synergistically to protect human MSCs from oxidative stress. 
A
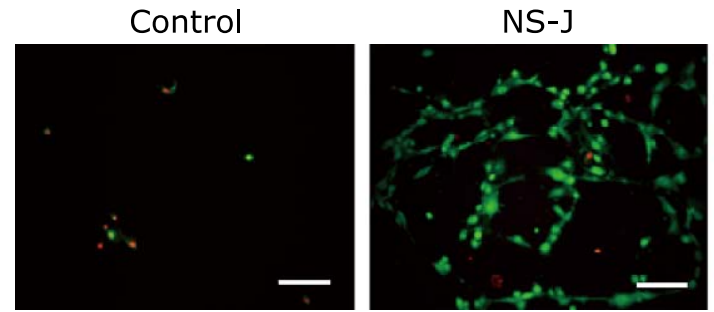

Ginger

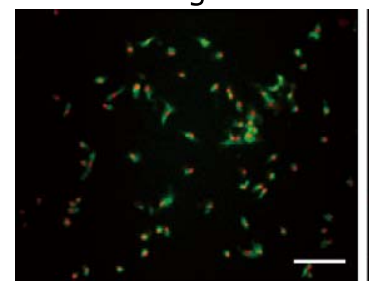

B

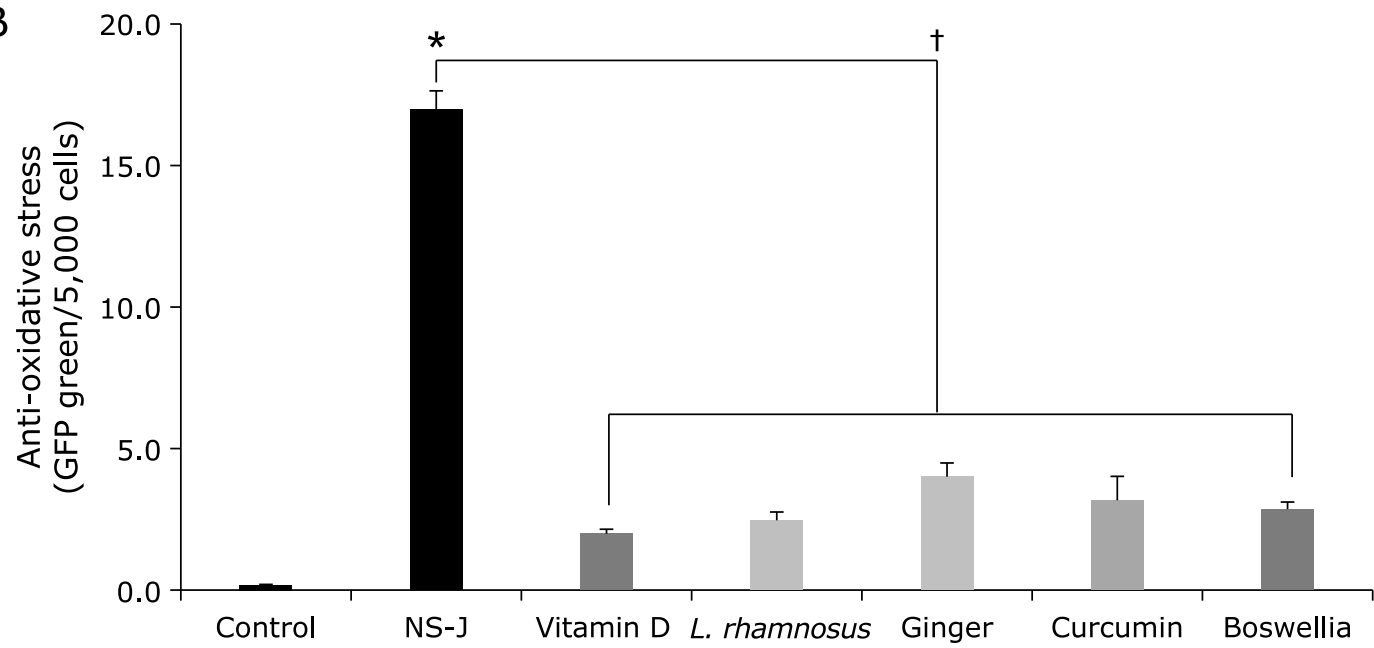

Fig. 1. Human MSCs were plated in a 24-well plate and $100 \mu \mathrm{M} \mathrm{H} \mathrm{H}_{2}$ was added to the cells followed by a 12-h incubation. Cell survival was measured by calcein AM staining. The concentration of all ingredients was $50 \mu \mathrm{g} / \mathrm{ml} .{ }^{*} p<0.05$ vs Control, ${ }^{\dagger} p<0.001$ vs NS-J. Scale bar $=100 \mu \mathrm{m}$. See color figure in the on-line version.

NS-J enhances the osteogenesis of hBM-MSCs. We next examined the effects of the natural supplements and the combined formula (NS-J) on osteogenic differentiation of hBM-MSCs Using calcium deposition in formed osteoblasts. Alizarin Red S-stained cells were visualized by phase-contrast microscopy to determine cell morphology and verify the presence of red mineralized nodules. The hBM-MSCs treated with individual natural ingredients did not form more osteoblasts than the control cells (Fig. 2A). By contrast, treatment with the combination formula drastically increased the formation of osteoblasts compared with treatment with individual components of the formula (Fig. 2B). These results indicate that synergism among the ingredients of NS-J amplifies the osteogenic effects of the individual ingredients.

NS-J enhances the chondrogenesis of hBM-MSCs.

Through chondrogenic differentiation, MSCs start to aggregate and condense, forming chondrocyte spheroids in vitro (Fig. 3A). Thus, we examined the effects of the natural ingredients and NS-J on chondrogenesis of hBM-MSCs by measuring chondrocyte spheroids. hBM-MSCs treated with Boswellia extract formed a few small spheroids. Cells treated with ginger, curcumin, or vitamin D formed larger chondrocyte spheroids and a few round spheroids. Cells treated with L. rhamnosus formed a great number of large spheroids than the control cells, and those spheroids produced a noticeable amount of glycosaminoglycans (GAGs). Cells treated with NS-J formed larger chondrocyte spheroids and generated a substantial number of surrounding GAGs. These results suggest that NS-J has a strong chondrogenic effect on human MSCs. We next quantified the amount of type II collagen, the major constituent of functional joint cartilage in chondrocytes, after treating cells with individual natural ingredients and NS-J. Compared with the control treatment and treatment with individual natural products, treatment with NS-J significantly increased production of type II collagen (Fig. 3B and C). These results demonstrate that vitamin $\mathrm{D}$ and L. rhamnosus have chondrogenic effects and that NS-J enhanced functional chondrogenesis in hBM-MSCs in a synergistic manner.

NS-J enhances chondrogenic differentiation under inflammatory conditions and increases the expression of PPAR- $\delta$.

We next examined whether the chondrogenic effects of NS-J were maintained under inflammatory conditions. hBM-MSCs (passages 2-3) were incubated in chondrogenic medium with and without HA, NS-J and 50\% human OA synovial fluid for 14 days. Under inflammatory conditions (presence of OA synovial fluid), chondrocyte spheroids were smaller than those formed in the absence of OA synovial fluid. By contrast, NS-J significantly increased the size of chondrocyte spheroids even in the presence of OA synovial fluid with no difference in size of chondrocyte 
A
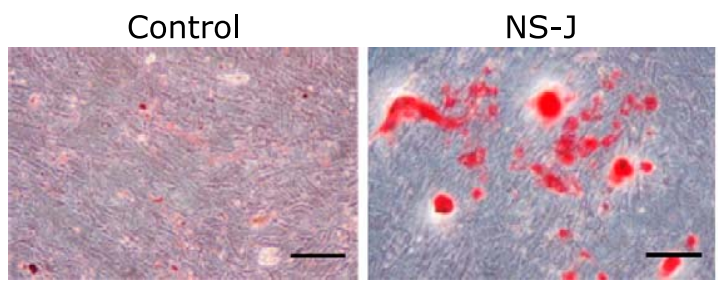

Ginger
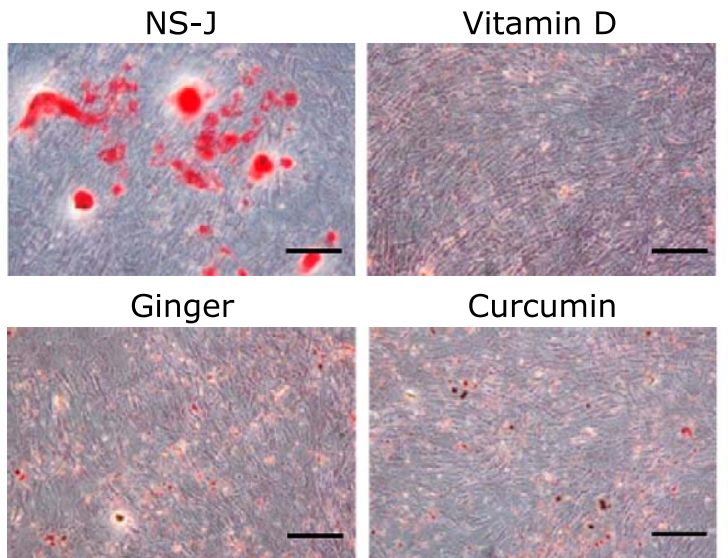

Curcumin

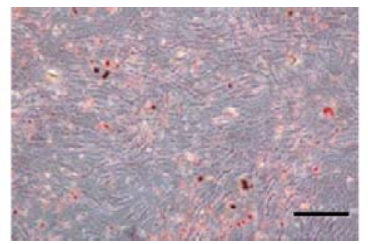

B

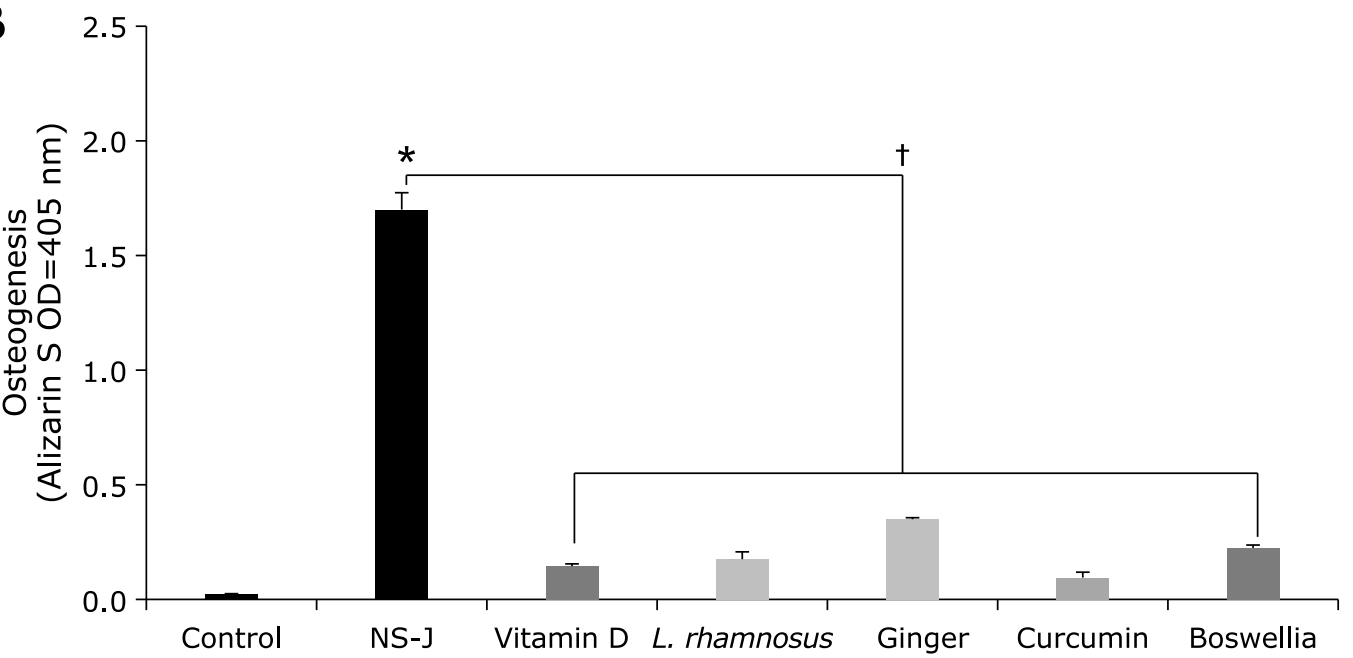

Fig. 2. (A) Alizarin Red S was used to stain human MSCs treated individual ingredients and NS-J. (B) Osteogenesis was quantified by Alizarin Red S staining of human MSCs. Human MSCs were incubated in osteogenic media. On day 17, cells were fixed and stained with Alizarin Red S. The concentration of all ingredients was $50 \mu \mathrm{g} / \mathrm{ml}$. ${ }^{*} p<0.05$ vs Control, ${ }^{\dagger} p<0.001$ vs NS-J. Scale bar $=100 \mu \mathrm{m}$. See color figure in the on-line version.

spheroids compared with the non-inflammatory control condition. HA had no significant effect on size of spheroids under inflammatory conditions (Fig. 3D). This result indicates that the antiinflammatory effect of NS-J overwhelmed the anti-chondrogenic effect of inflammation.

NS-J showed properties similar to those of a PPAR- $\delta$ agonist (GW0742), includin anti-inflammatory, pro-chondrogenic and anti-adipogenic effects. ${ }^{(25)}$ We therefore used immunoblotting to examine the expression of type II collagen and PPAR- $\delta$ in response to NS-J treatment. Inflammatory conditions decreased the expression of type II collagen and PPAR- $\delta$ whereas NS-J increased this expression under inflammatory conditions. HA had no significant effect on protein expression (Fig. 3D-G). These results demonstrate that the properties of NS-J correlate with the PPAR- $\delta$ pathway, and that NS-J has anti-inflammatory effect. This is the first time that this natural supplement complex has been reported to affect the expression of PPAR $-\delta$.

NS-J decreases the adipogenesis of hBM-MSCs. To examine the effects of NS-J and its component ingredients on MSC adipogenesis hBM-MSCs were incubated in adipogenic medium with or without $50 \mu \mathrm{g} / \mathrm{ml}$ of NS-J for 14 days and then stained with Oil Red O, which stains lipid droplets. Images of lipid droplet-containing adipocytes that had differentiated from hBM-MSCs were captured under a light microscope. The extent of adipogenesis (accumulation of red lipid droplets) was measured using a microplate reader (absorbance at $490 \mathrm{~nm}$ ). NS-J significantly reduced adipogenesis of hBM-MSCs (Fig. 4A and B).

\section{Discussion}

Oxidative stress causes degeneration and apoptosis of cells including MSCs, in various organ tissues, thereby contributing to inflammatory, metabolic, and degenerative diseases. ${ }^{(26)} \mathrm{We}$ examined whether the combination of several natural products could protect hBM-MSCs from oxidative stress caused by $\mathrm{H}_{2} \mathrm{O}_{2}$. Although some individual components of the formula offered some protection, the combination of vitamin D, L. rhamnosus, ginger, curcumin and Boswellia extract (NS-J) greatly enhanced the survival rate of human MSCs exposed to oxidative stress. The antioxidant properties of the individual components appeared to contribute slightly to protection, but the extent of protection was greater than an additive effect. This combination shows synergism on the antioxidant effect.

Further experiments revealed that NS-J also had synergistic effects on human MSCs in terms of decreased adipogenesis and enhanced osteogenesis, and chondrogenesis. L. rhamnosus by itself had a considerable anti-adipogenic effect, but NS-J reduced MSC adipogenesis even furthers. The anti-adipogenic effects of 
A

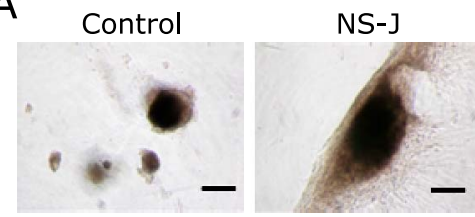

Ginger

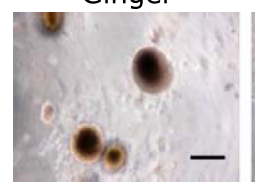

C

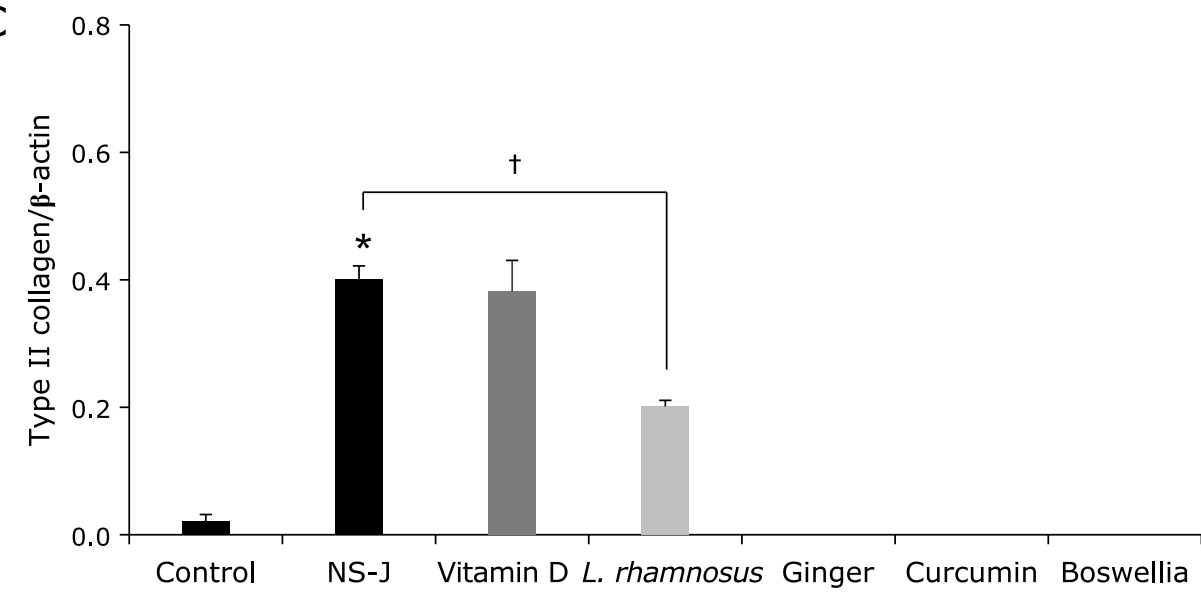

B

Type II collagen

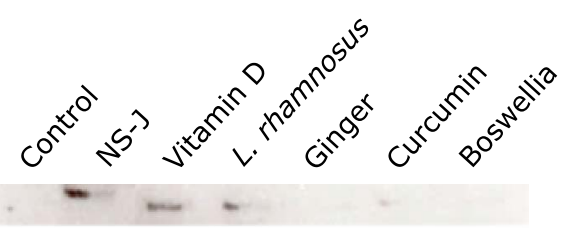

$\boldsymbol{\beta}$-actin
D

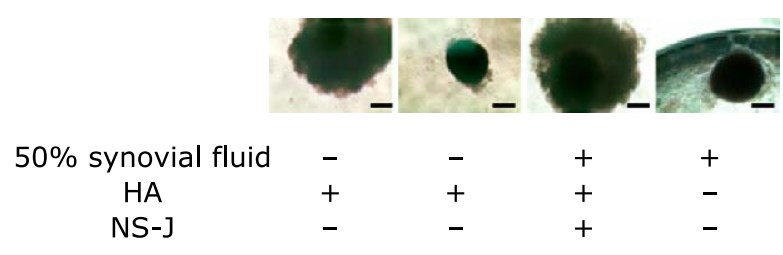

E

Type II collagen

Control SF SF+NS-J No HA

PPAR- $\delta$

$\beta$-actin

$50 \%$ synovial fluid

HA

NS-J
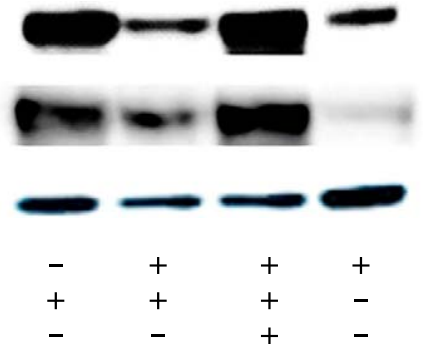

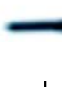

$$
+
$$

- $\quad-$
F

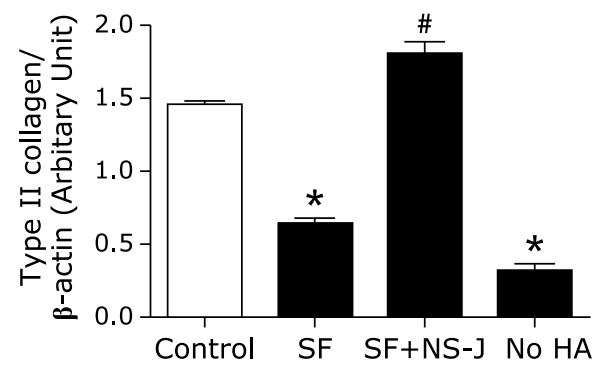

G

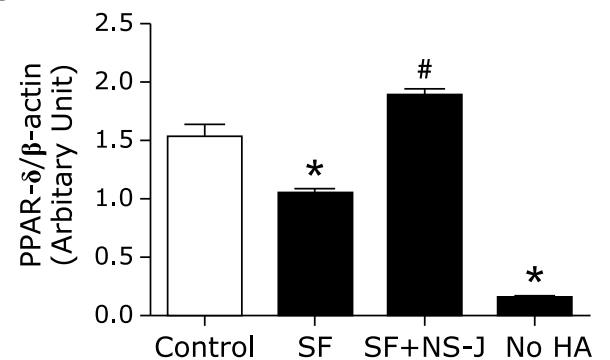

Fig. 3. (A) Human MSCs were cultured in $70 \mu \mathrm{l}$ of clinical hyaluronic acid (HA) gel during chondrogenesis in 96-well plates. (B) Human MSCs were cultured in $70 \mu \mathrm{l}$ of Euflexxa ${ }^{\circledR}$ during chondrogenesis in 96-well plates. Relative levels of type II collagen and $\beta$-actin (C) were quantified by immunoblotting and results are presented in bar graphs. MSCs were incubated in chondrogenic media. Chondrocyte hyaline was measured by assessing expression of type II collagen. (D) Human MSCs were cultured with $50 \%$ human osteoarthritis synovial fluid for 14 days to compare chondrogenic effect of natural supplement under inflammatory condition. (E-G) The expression of type II collagen and PPAR- $\delta$ were quantified by immunoblotting ${ }^{*} p<0.05$ vs Control, ${ }^{+} p<0.001$ vs NS-J, ${ }^{*} p<0.05$ vs SF. Scale bar $=100 \mu \mathrm{m}$. See color figure in the on-line version. 

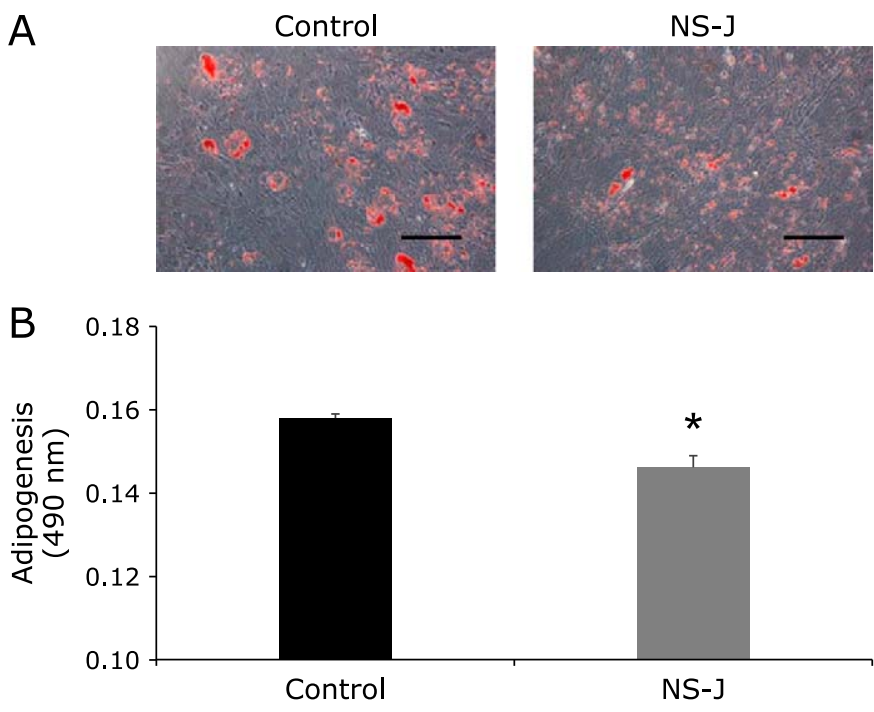

Fig. 4. (A) Human MSCs were incubated in adipogenic media. After 14-day incubation, cells were fixed and stained with Oil Red $O$ to stain lipid droplets. The concentration of NS-J was $50 \mu \mathrm{g} / \mathrm{ml}$. ${ }^{*} p<0.05$ vs Control. Scale bar $=100 \mu \mathrm{m}$. See color figure in the on-line version.

the individual components likely contributed to the synergistic anti-adipogenic effect of NS-J, but most of the anti-adipogenic effect, was likely contributed by $L$. rhamnosus, consistent with the finding of a previous study. ${ }^{(14)}$ The anti-adipogenic effect of NS-J on human MSCs is a new finding of this study.

NS-J had significant synergistic effects in osteogenesis. Chondrogenesis was also enhanced by NS-J. NS-J treatment generated the most noticeable extracellular matrix around chondrocyte spheroids and a larger amount of type II collagen than the other treatments tested. Vitamin D and L. rhamnosus had significant chondrogenic effects, and ginger, curcumin and Boswellia extract each had a significant osteogenic effect. Ginger and curcumin had a significant anti-oxidative effect. The combination of these natural supplements produced synergistic interactions among the individual components.

Oxidation regulates the pathway of osteogenesis, chondrogenesis and adipogenesis. ${ }^{(27)}$ Reactive oxygen species (ROS) are essential in the early stage of chondrogenic differentiation. ${ }^{(28)}$ Chen et al. ${ }^{(29)}$ reported that ROS inhibit osteogenic differentiation of MSCs. Those studies support our finding that the anti-oxidative effect of NS-J affects the osteogenesis, chondrogenesis and adipogenesis of MSCs. Synergism in the anti-oxidative effect also affects the synergism of NS-J's other properties.

A previous study reported that a mixture of a PPAR- $\delta$ agonist (GW0742), MSCs and HA (CHAMP) had anti-inflammatory, pro-chondrogenic and anti-adipogenic effects. ${ }^{(25)}$ The properties reported for the PPAR- $\delta$ agonist mixture were similar to those we observed for NS-J. We therefore demonstrated a correlation between PPAR- $\delta$ and NS-J. The data which shows that NS-J increased the expression of PPAR- $\delta$, suggest that the antiinflammatory, anti-adipogenic and pro-chondrogenic effects of NS-J are mediated through the PPAR- $\delta$ pathway. This hypothesis could explain the medical effects of this combination of natural supplements. A solid understanding of the mechanism of natural supplements could be used to reinforce their medical effects and inhibit side effects.

Most of the individual components of NS-J have anti-arthritic effects as well as analgesic and anti-inflammatory effects, ${ }^{(7,9,15,17,22)}$ and the combined formula is ideal for treating joint arthritis as well as osteoarthritis because NS-J enhances chondrogenesis and reduces pain and inflammation. Several studies report that combinations of phytomedicines have synergistic effects. ${ }^{(30,31)}$ NS-J demonstrated synergistic benefits that could help treat arthritis. Because the pharmacokinetics of each component are likely different, their synergistic effects could vary in different tissues, which remains to be explored in future studies.

A limitation of our study is that although the anti-inflammatory and analgesic effects of the individual components of NS-J have been studied, ${ }^{(7,9,15,17,22)}$ the synergistic interactions among them have not yet been analyzed. In addition, further research is required to determine the molecular mechanisms by which the combination of ingredients had anti-oxidative, osteogenic, chondrogenic and anti-adipogenic effects. Additional molecular mechanism studies are also needed to explain the synergistic effect.

In summary, we demonstrated that NS-J promotes differentiation of MSCs into osteoblasts or chondrocytes to a greater extent than its individual ingredients, indicating synergistic interactions among those ingredients. In addition, NS-J protected MSCs from oxidative stress. These effects of NS-J likely occur through stimulation of the PPAR- $\delta$ signaling pathway. The chondrogenic effects of NS-J could promote healthy cartilage and reduce the pain and symptoms of osteoarthritis.

\section{Author Contributions}

DHK (Dong Hwan Kim) and DHK (Dong Hyun Kim): collection and assembly of data, data analysis and interpretation, manuscript writing; BEH and MS: collection and assembly of data and data analysis; JH and KHY: conception and design, manuscript writing, data analysis and interpretation, final approval of manuscript.

\section{Acknowledgments}

This investigation was supported by NWO Stem Cure, LLC. This work was also supported by the Ministry of Health \& Welfare, Republic of Korea (Grant No. HI15C2963) and the National Research Foundation of Korea (NRF; Grant NRF 2019R1I1A1A01063186). J.H is supported by the Basic Science Research Program of the NRF (2017R1A2B2012305).

\section{Conflict of Interest}

This study was funded by NWO Stem Cure, LLC. None of the authors have potential conflicts of interest with respect to the research, authorship, and/or publication of this article.

\section{References}

1 Grover AK, Samson SE. Benefits of antioxidant supplements for knee osteoarthritis: rationale and reality. Nutr J 2016; 15: 1.

2 William BM, Brillhart K, Afable M, et al. A phase II study of curcumin and vitamin $\mathrm{D}$ in previously untreated patients with early stage chronic lymphocytic leukemia (CLL) or small lymphocytic lymphoma (SLL). Blood 2018; 132 (Suppl 1): 1875.

3 Jerzynska J, Stelmach W, Balcerak J, et al. Effect of Lactobacillus rhamnosus GG and vitamin D supplementation on the immunologic effectiveness of

grass-specific sublingual immunotherapy in children with allergy. Allergy Asthma Proc 2016; 37: 324-334.

4 Deol PK, Khare P, Bishnoi M, Kondepudi KK, Kaur IP. Coadministration of ginger extract-Lactobacillus acidophilus (cobiotic) reduces gut inflammation and oxidative stress via downregulation of $C O X-2, i-N O S$, and $c-M y c$. Phytother Res 2018; 32: 1950-1956.

5 Nicolson GL. Mitochondrial dysfunction and chronic disease: treatment with natural supplements. Altern Ther Health Med 2014; 20 Suppl 1: 18-25. 
6 Lee JY, Harvey WF, Price LL, Paulus JK, Dawson-Hughes B, McAlindon TE. Relationship of bone mineral density to progression of knee osteoarthritis. Arthritis Rheum 2013; 65: 1541-1546.

7 Tetlow LC, Woolley DE. Expression of vitamin D receptors and matrix metalloproteinases in osteoarthritic cartilage and human articular chondrocytes in vitro. Osteoarthritis Cartilage 2001; 9: 423-431.

8 Ryan LM, Teach SJ, Singer SA, et al. Bone mineral density and vitamin D status among African American children with forearm fractures. Pediatrics 2012; 130: e553-e560.

9 Vaghef-Mehrabany E, Alipour B, Homayouni-Rad A, Sharif SK, AsghariJafarabadi M, Zavvari S. Probiotic supplementation improves inflammatory status in patients with rheumatoid arthritis. Nutrition 2014; 30: 430-435.

10 Taverniti V, Guglielmetti S. The immunomodulatory properties of probiotic microorganisms beyond their viability (ghost probiotics: proposal of paraprobiotic concept). Genes Nutr 2011; 6: 261-274.

11 Ammoscato F, Scirocco A, Altomare A, et al. Lactobacillus rhamnosus protects human colonic muscle from pathogen lipopolysaccharide-induced damage. Neurogastroenterol Motil 2013; 25: 984-e777.

12 Lin PW, Myers LE, Ray L, et al. Lactobacillus rhamnosus blocks inflammatory signaling in vivo via reactive oxygen species generation. Free Radic Biol Med 2009; 47: 1205-1211.

13 Grompone G, Martorell P, Llopis S, et al. Anti-inflammatory Lactobacillus rhamnosus CNCM I-3690 strain protects against oxidative stress and increases lifespan in Caenorhabditis elegans. PLoS One 2012; 7: e52493.

14 Kim SW, Park KY, Kim B, Kim E, Hyun CK. Lactobacillus rhamnosus GG improves insulin sensitivity and reduces adiposity in high-fat diet-fed mice through enhancement of adiponectin production. Biochem Biophys Res Commun 2013; 431: 258-263.

15 Ribel-Madsen S, Bartels EM, Stockmarr A, et al. A synoviocyte model for osteoarthritis and rheumatoid arthritis: response to Ibuprofen, betamethasone, and ginger extract-a cross-sectional in vitro study. Arthritis 2012; 2012: 505842 .

16 Mashhadi NS, Ghiasvand R, Askari G, Hariri M, Darvishi L, Mofid MR. Anti-oxidative and anti-inflammatory effects of ginger in health and physical activity: review of current evidence. Int J Prev Med 2013; 4 (Suppl 1): S36S42.

17 Kuptniratsaikul V, Dajpratham P, Taechaarpornkul W, et al. Efficacy and safety of Curcuma domestica extracts compared with ibuprofen in patients with knee osteoarthritis: a multicenter study. Clin Interv Aging 2014; 9: 451458.
18 Valentine C, Ohnishi K, Irie K, Murakami A. Curcumin may induce lipolysis via proteo-stress in Huh7 human hepatoma cells. J Clin Biochem Nutr 2019; 65: $91-98$.

19 Buhrmann C, Mobasheri A, Matis U, Shakibaei M. Curcumin mediated suppression of nuclear factor- $\kappa \mathrm{B}$ promotes chondrogenic differentiation of mesenchymal stem cells in a high-density co-culture microenvironment. Arthritis Res Ther 2010; 12: R127.

20 Davis JM, Murphy EA, Carmichael MD, et al. Curcumin effects on inflammation and performance recovery following eccentric exercise-induced muscle damage. Am J Physiol Regul Integr Comp Physiol 2007; 292: R2168R2173.

21 Kimmatkar N, Thawani V, Hingorani L, Khiyani R. Efficacy and tolerability of Boswellia serrata extract in treatment of osteoarthritis of knee--a randomized double blind placebo controlled trial. Phytomedicine 2003; 10: 3-7.

22 Sengupta K, Alluri KV, Satish AR, et al. A double blind, randomized, placebo controlled study of the efficacy and safety of 5-Loxin for treatment of osteoarthritis of the knee. Arthritis Res Ther 2008; 10: R85.

23 Ammon HP. Modulation of the immune system by Boswellia serrata extracts and boswellic acids. Phytomedicine 2010; 17: 862-867.

24 Kota BP, Huang TH, Roufogalis BD. An overview on biological mechanisms of PPARs. Pharmacol Res 2005; 51: 85-94.

25 Heck BE, Park JJ, Makani V, Kim EC, Kim DH. PPAR- $\delta$ agonist with mesenchymal stem cells induces type II collagen-producing chondrocytes in human arthritic synovial fluid. Cell Transplant 2017; 26: 1405-1417.

26 Chandra J, Samali A, Orrenius S. Triggering and modulation of apoptosis by oxidative stress. Free Radic Biol Med 2000; 29: 323-333.

27 Yanes O, Clark J, Wong DM, et al. Metabolic oxidation regulates embryonic stem cell differentiation. Nat Chem Biol 2010; 6: 411-417.

28 Chen CT, Shih YR, Kuo TK, Lee OK, Wei YH. Coordinated changes of mitochondrial biogenesis and antioxidant enzymes during osteogenic differentiation of human mesenchymal stem cells. Stem Cells 2008; 26: 960-968.

29 Kim KS, Choi HW, Yoon HE, Kim IY. Reactive oxygen species generated by NADPH oxidase 2 and 4 are required for chondrogenic differentiation. $J$ Biol Chem 2010; 285: 40294-40302.

30 Williamson EM. Synergy and other interactions in phytomedicines. Phytomedicine 2001; 8: 401-409.

31 Yang Y, Zhang Z, Li S, Ye X, Li X, He K. Synergy effects of herb extracts: pharmacokinetics and pharmacodynamic basis. Fitoterapia 2014; 92: 133147. 